\title{
Effective strategy in the treatment of aortobronchial fistula with recurrent hemoptysis
}

\author{
Shin-Ah Son, Deok Heon Lee, Gun-Jik Kim \\ Department of Thoracic and Cardiovascular Surgery, School of Medicine, Kyungpook National University, Kyungpook National University \\ Hospital, Daegu, Korea
}

Received: December 30, 2019

Revised: February 3, 2020

Accepted: February 6, 2020

Corresponding author:

Gun-Jik Kim

Department of Thoracic and

Cardiovascular Surgery, School of

Medicine, Kyungpook National

University, Kyungpook National

University Hospital, 130 Dongdeok-

ro, Jung-gu, Daegu 41944, Korea

Tel: +82-53-200-5665

Fax: +82-53-426-4765

E-mail: straightroot@knu.ac.kr
Aortobronchial fistula (ABF) involves the formation of an abnormal connection between the thoracic aorta and the central airways or the pulmonary parenchyma and is associated with an increased risk of mortality. An ABF typically manifests clinically with symptoms of hemoptysis, and currently, there is a lack of defined guidelines for its treatment. Here, we report the cases of two patients who suffered from recurrent hemoptysis due to ABF with pseudoaneurysm. We propose that removal of the aorta with concomitant lung resection and coverage of the aorta using the pericardial membrane is a definite treatment to lower recurrence of $A B F$ and persistent infection.

Keywords: Aorta; Endovascular procedure; Hemoptysis; Surgery

\section{Introduction}

Aortobronchial fistula $(\mathrm{ABF})$ is a rare and devastating complication if left untreated. It involves the formation of an abnormal connection between the thoracic aorta and the central airways or the pulmonary parenchyma and results in fatal complications [1]. With the increasing use of thoracic endovascular repair (TEVAR), the number of patients treated for $\mathrm{ABF}$ has increased $[2,3]$. Most $\mathrm{ABFs}$ occur in ill patients; therefore, there is no determined treatment. Conservative non-surgical therapy can result invariably in a fatal outcome from massive hemoptysis or chronic mediastinitis [4], and stent-graft insertion is safe and less invasive. However, there are limitations, and generally there is a higher risk of graft infection with repeated ABFs $[4,5]$. Here, we present patients with ABFs that have been successfully treated by removal of the aorta, concomitant resection of the lung, and coverage of the aorta using the pericardial membrane to prevent further erosive damage.

\section{Cases}

\section{Patient consent}

All authors declare that written informed consent was obtained from the patient for publication of this case report and accompanying images.

\section{Case 1}

A 65-year-old man visited an outpatient clinic with a complaint of recurrent hemoptysis. The patient was diagnosed with $\mathrm{ABF}$ with pseudoaneurysm 4 years previously (Fig. 1A) and underwent TEVAR $(42 \times 38 \times 150 \mathrm{~mm}, 40 \times 40 \times 130 \mathrm{~mm}$; S\&G Biotech, Seongnam, Korea). Two years post-TEVAR, the patient presented with recurrent episodes of hemoptysis and chills during a routine follow-up visit.

Chest computed tomography (CT) imaging revealed a newly formed type 1a endoleak and an even larger pseudoaneurysm at

Copyright(C) 2020 Yeungnam University College of Medicine

This is an Open Access article distributed under the terms of the Creative Commons Attribution Non-Commercial License (http://creativecommons.org/licenses/by-nc/4.0/) which permits unrestricted non-commercial use, distribution, and reproduction in any medium, provided the original work is properly cited. 
the previous stent insertion site (Fig. 1B). To rectify these problems, a hybrid debranching TEVAR was performed on the innominate artery and left common carotid artery $(40 \times 40 \times 200$ $\mathrm{mm}$; S\&G Biotech) via a medial sternotomy. After the second TEVAR, several episodes of sudden hemoptysis occurred that were initially negligible, but the symptoms gradually became severe. The patient was hemodynamically stable; however, mild signs of a systemic inflammatory response were noted. In the emergency department, chest CT revealed a type $1 \mathrm{~b}$ endoleak from the previous stent-graft with progression of the pseudoaneurysm and lung consolidation with atelectasis (Fig. 1C, 1D).
The patient underwent surgery using extracorporeal circulation via femorofemoral cardiopulmonary bypass (CPB). An approach was made through the 4th and 7 th intercostal spaces for proximal and distal graft anastomosis site manipulation, respectively. After removal of the lung adhesion, an opening was made in the pseudoaneurysm. The cut revealed a dark hematoma and inflammatory tissue within the vessel. Dissection between the visceral pleura and the aorta seemed unfeasible due to the large degree of adhesion. The presence of extensive lung hemorrhage and necrotic change led to the decision to entirely remove the affected lesion. Stent-graft removal, 30-mm Hemashield graft replacement, and
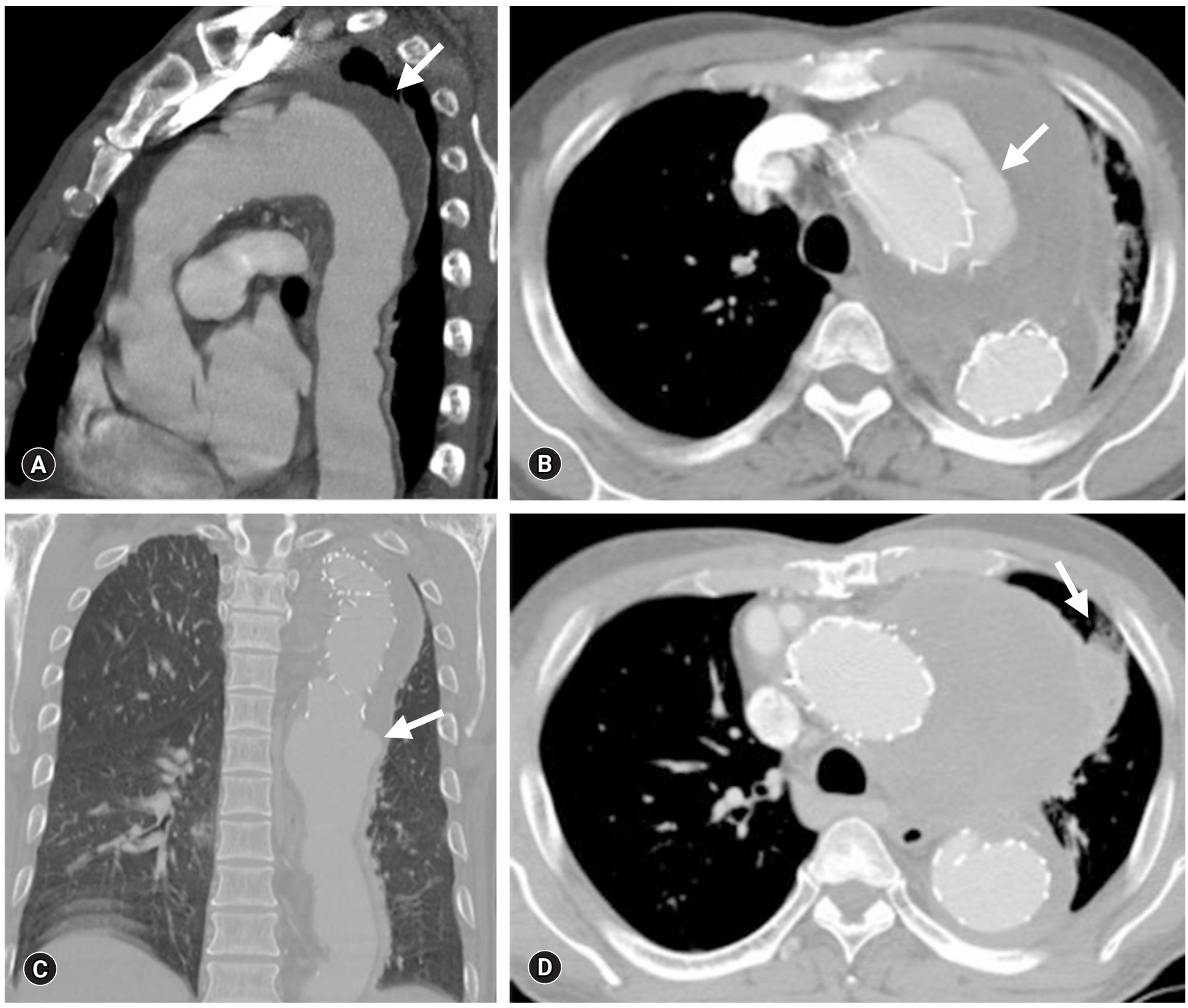

Fig. 1. Chest computed tomography (CT) findings. (A) Initial chest CT shows descending aortic pseudoaneurysm (arrow). (B) Two years later, chest CT reveals a newly formed type 1a endoleak (arrow) and an even larger pseudoaneurysm. (C) Chest CT on admission reveals type $1 \mathrm{~b}$ endoleak (arrow) from the previous stent with progression of the pseudoaneury sm. (D) Lung consolidation with atelectasis (arrow). 
left apicoposterior-anterior segmentectomy were performed simultaneously. As a preventive measure, space between the stentgraft and pulmonary tissue was packed with the pericardial membrane. The patient's condition gradually improved, and he was discharged on postoperative day (POD) 22 (Fig. 2).

\section{Case 2}

A 66-year-old man was admitted to our medical center with massive and recurrent hemoptysis over a 4-year period. The patient was initially diagnosed with $\mathrm{ABF}$ with pseudoaneurysm and had undergone TEVAR $(38 \times 38 \times 150 \mathrm{~mm}$; Valiant Thoracic Stent Graft, Medtronic CardioVascular, Santa Rosa, CA, USA) (Fig. 3A). Two years post-TEVAR, the patient visited our medical center's emergency room with another episode of hemoptysis. A kinking stenosis of the aortic isthmus stent-graft was discovered via chest CT, and a new stent-graft $(40 \times 40 \times 80 \mathrm{~mm}$, Valiant Thoracic Stent Graft) was inserted (Fig. 3B).

Furthermore, the patient had displayed malaise, chills, and recurrent hemoptysis at admission; however, there were no clinical or biological signs of infectious disease, and vital signs were stable. There was no evidence of endoleaks via chest CT, but air entrapment within the thrombosed aneurysm and lung consolidation were found (Fig. 3C). Bronchoscopy revealed oozing at the left upper lobe orifice (Fig. 3D).

Due to the possibility of infection, we decided to perform open surgery to remove the $\mathrm{ABF}$ causing the hemoptysis. An initial incision was made in the $3 \mathrm{rd}$ or 4 th intercostal space with the assistance of CPB. There was a severe adhesion between the previously inserted stent-graft and the left upper lobe. Upon exposing the pseudoaneurysm, the affected stent-graft was removed, and a 30- mm Hemashield graft was inserted as a replacement. The left lower lung was relatively well-preserved; however, an apicoposterior segment of the left upper lung was suspected of fistula formation. A left upper lobectomy was performed with coverage of the aorta using the pericardial membrane. After administration of adequate antibiotic therapy for 8 weeks, the patient was discharged after POD 30 with oral antibiotics (Fig. 4).

\section{Discussion}

$\mathrm{ABF}$ is a rare diagnosis and has a relatively low incidence, ranging from $0.56 \%$ to $1.7 \%[1,2,6]$. There are several causes of ABF with persistent or newly developed endoleaks playing a crucial role after TEVAR [1]. The postulated cause of ABF post-TEVAR includes stent-graft coverage of the bronchial arteries leading to ischemic necrosis of the bronchial wall due to stent coverage of the feeding arteries, chronic endoleaks leading to erosion into the adjacent lung, and penetration of the stent-graft through the aortic wall into the lung [7]. In recent years, there has been an increase in the number of patients diagnosed and treated for $\mathrm{ABF}$ post-TEVAR $[1,7,8]$. Pseudoaneurysm as an indication for TEVAR represents a significant risk factor for ABF. Compression of the airways by the pseudoaneurysm may result in a local inflammatory response and the formation of stable adhesions and tissue necrosis, leading to erosion and finally fistulization [2]. In addition, longstanding atelectasis may indicate adherence to the aneurysmal aortic wall that is already lacking elasticity, and external compression of the bronchial tree might also occur [8].

Clinical presentation of $\mathrm{ABF}$ can be a display of hemoptysis at any degree $[5,9,10]$. A diagnosis is primarily determined by CT,
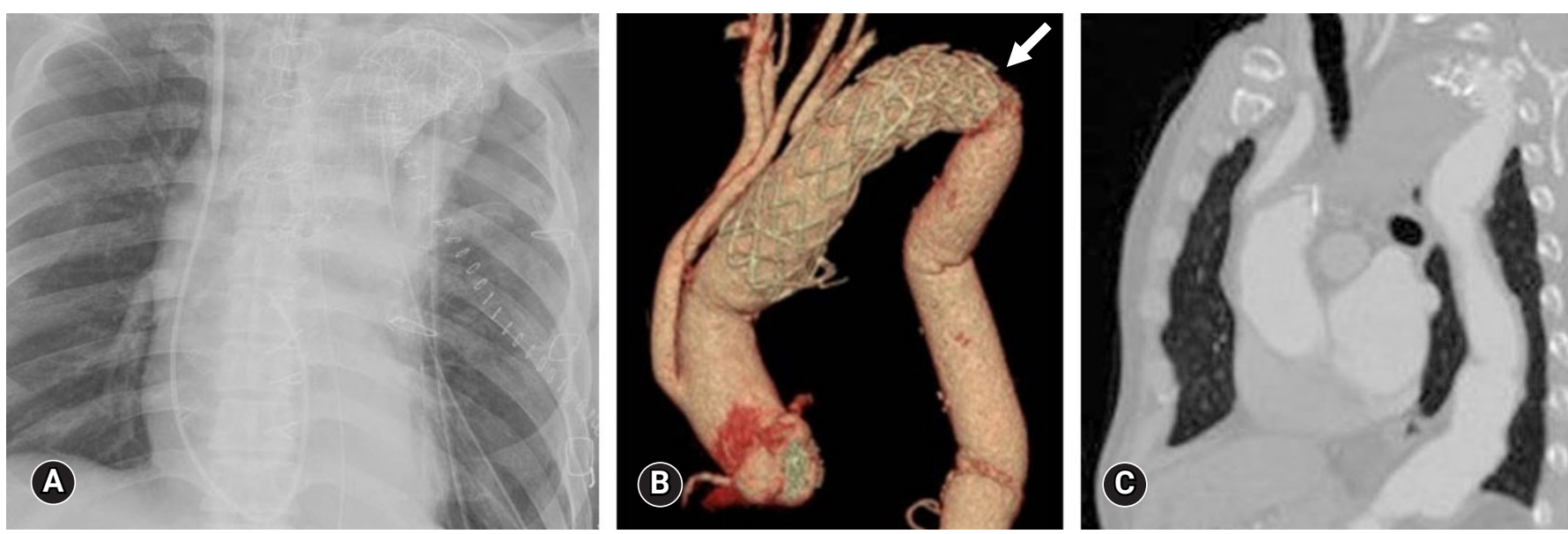

Fig. 2. Postoperative chest radiograph and computed tomography (CT) findings of case 1. (A) Postoperative chest radiograph indicates a well-functioning Hemashield graft. (B) Postoperative chest CT indicates the absence of endoleaks (arrow). (C) Postoperative chest CT confirms the absence of a lung lesion. 

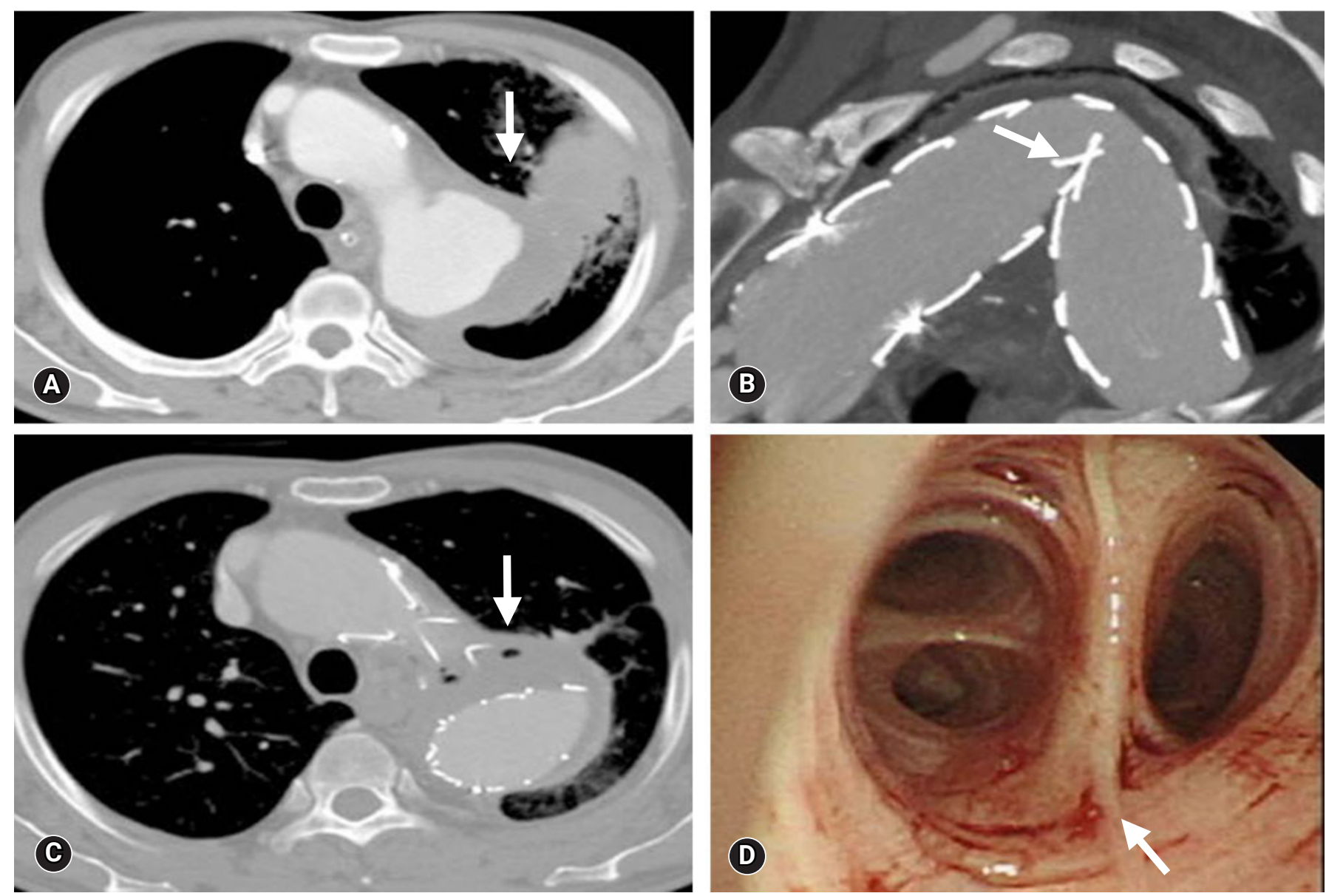

Fig. 3. Chest computed tomography (CT) findings of case 2. (A) Initial chest CT indicates a pseudoaneurysm with a suspicious aortobronchial fistula lesion (arrow). (B) A follow-up chest CT reveals kinking stenosis of the aortic isthmus stent-graft (arrow). (C) Chest CT on admission reveals air entrapment within the thrombosed aneurysm (arrow). (D) Brochoscopy shows oozing at left upper lobe orifice (arrow).
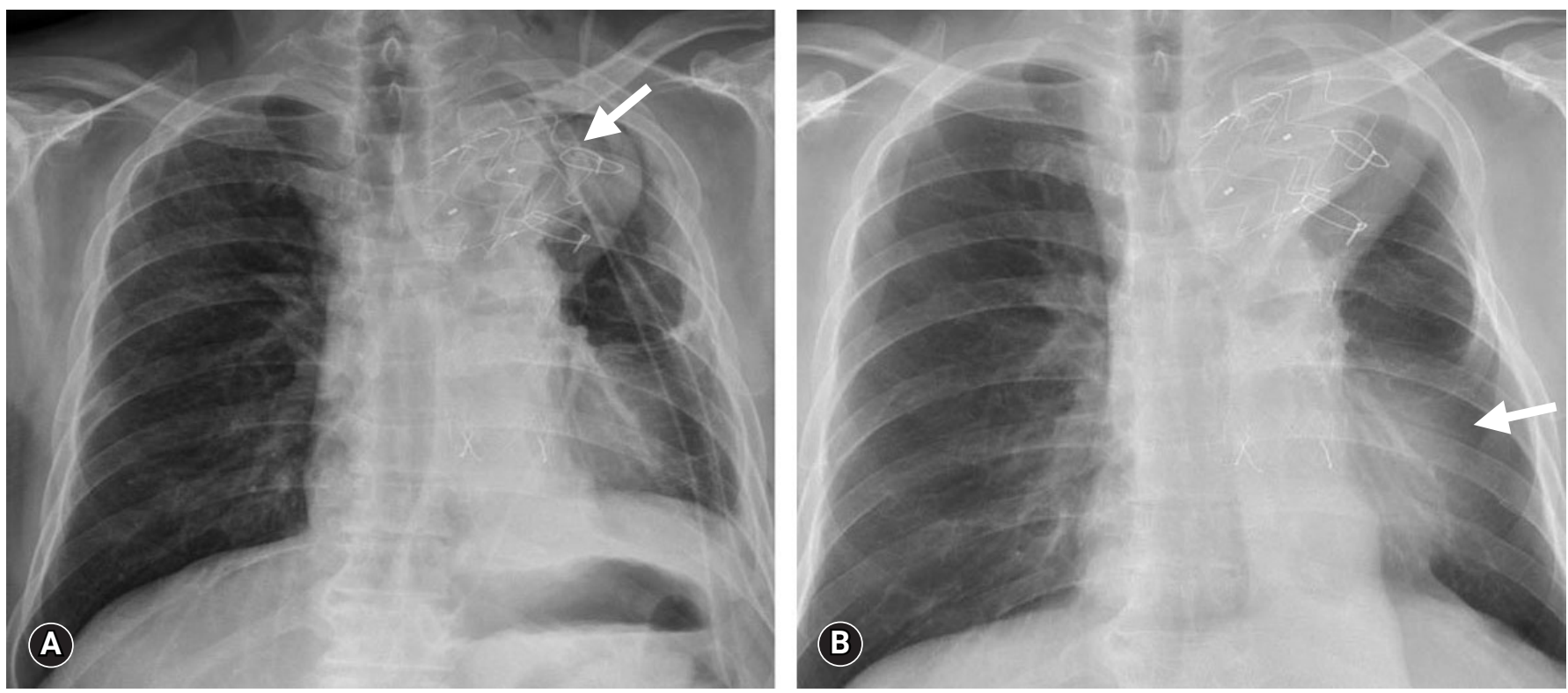

Fig. 4. Postoperative chest radiograph findings of case 2. (A) Postoperative chest radiograph shows remnant stent-graft (arrow). (B) Postoperative chest radiograph (followed-up after 4 years) shows the abscence of lung lesion (arrow). 
with CT angiography being considered the first diagnostic imaging study performed in the majority of cases due to its ease of use in emergency situations. Although CT rarely detects fistulous tracts, suggestive signs of $\mathrm{ABF}$ are present in most patients, which include air entrapment within the thrombosed aneurysm, periaortic fluid collection, bronchial wall thickening, and lung consolidation $[11,12]$.

The most important factor in the prognosis of $\mathrm{ABF}$ patients is the presence of infection. Once infection has occurred within the vessel, it readily extends to the bronchial wall and lung parenchyma, which can ultimately lead to sepsis. When infection of the stent-graft or pseudoaneurysm is suspected, the majority of cases will require repair via open surgery [13]. Although surgical principles dictate control of infection, removal of all infected tissues and reconstruction in a clean field is not always achievable, as it places a huge demand on an already sick patient [13]. Moreover, operative mortality was approximately $50 \%$ in patients with infection $[6,14]$.

The principles of treatment of ABFs are based on the condition of the patient. Conservative non-surgical therapy with antibiotic treatment is used in patients that do not consent to invasive treatment due to low levels of hemoptysis and/or multiple comorbidities. However, conservative non-surgical therapy results invariably in a fatal outcome as a result of massive hemoptysis or chronic mediastinitis [4]. Endovascular repair followed by antibiotic therapy is described in the treatment of $\mathrm{ABF}$, particularly in cases unfit for open surgical treatment [15]. Within the last decade, several case reports have described initial success with treatment of $\mathrm{ABF}$ using stent-graft insertion, suggesting more widespread use of this approach. The perioperative outcomes of endovascular repair have been favorable with a low 30day mortality rate (6.4\%) [5]. It is uncertain whether endovascular repair alone provides a complete and reliable cure for an $\mathrm{ABF}$ as endovascular repair can result in risk of recurrence or stentgraft infection [4]. From these findings, direct contact between the involved aorta and the pulmonary tissue should be avoided to prevent further erosive damage and subsequent infection. Open $\mathrm{ABF}$ repair is associated with a high mortality rate, reported at $15 \%$ to $41 \%$, related to the need for thoracotomy, thoracic aortic cross-clamping, and the surgical replacement or repair of the thoracic aorta with concomitant resection of the pulmonary segments $[2,4,9,14]$.

In our cases, the most important factor for determining the cause of the $\mathrm{ABF}$ was the formation of a large pseudoaneurysm. If we considered more invasive surgical intervention initially or followed stricter indications for TEVAR, we could have avoided multiple procedures. From the operative findings, severe necrosis and inflammation of the lung and periaortic tissue were present in contrast to the mild symptoms of the patient. The presence of necrotic tissue increased the risk of infection, and we thought the presence of infection was critical to prognosis. To reduce the incidence of long-term persistent infection, we performed concomitant stent-graft removal and necrotic lung resection.

In the case of recurrent hemoptysis, $\mathrm{ABF}$ should be managed unconditionally to prevent devastating complications, and either elective or emergent open repair should be determined according to the patient's symptoms and hemodynamics. The risk of infection and recurrent $\mathrm{ABF}$ is high if the bronchial lesion is left untreated; therefore, a TEVAR-only approach is not advisable [5]. To reduce the incidence of long-term persistent infection and recurrent $\mathrm{ABF}$, removal of the aorta and necrotic lung tissue must be performed simultaneously.

This report highlights the utility and benefits of a surgical approach to resolve $\mathrm{ABF}$ and potentially eliminate infected lesions. On the basis of our experience with these cases, we would like to emphasize the importance of $\mathrm{ABF}$ management in pseudoaneurysm patients and recommend a surgical approach that involves total resection of the affected area as a guideline treatment for $\mathrm{ABF}$ with pseudoaneurysm.

\section{Acknowledgments}

\section{Conflicts of interest}

No potential conflict of interest relevant to this article was reported.

\section{Author contribution}

Conceptualization: SAS; Methodology: DHL, GJK; Resources: SAS; Writing-original draft, SAS; Writing-review \& editing: DHL, GJK.

\section{ORCID}

Shin-Ah Son, https://orcid.org/0000-0003-3317-6857

Deok Heon Lee, https://orcid.org/0000-0001-6206-4745

Gun-Jik Kim, https://orcid.org/0000-0002-8051-2131

\section{References}

1. Czerny M, Reser D, Eggebrecht H, Janata K, Sodeck G, Etz C, et al. Aorto-bronchial and aorto-pulmonary fistulation after thoracic endovascular aortic repair: an analysis from the European Registry of Endovascular Aortic Repair Complications. Eur J Cardiothorac Surg 2015;48:252-7.

2. Chiesa R, Melissano G, Marone EM, Marrocco-Trischitta 
MM, Kahlberg A. Aorto-oesophageal and aortobronchial fistulae following thoracic endovascular aortic repair: a national survey. Eur J Vasc Endovasc Surg 2010;39:273-9.

3. Luehr M, Etz CD, Nozdrzykowski M, Garbade J, Lehmkuhl L, Schmidt A, et al. Emergency open surgery for aorto-oesophageal and aorto-bronchial fistulae after thoracic endovascular aortic repair: a single-centre experience. Eur J Cardiothorac Surg 2015;47:374-82.

4. Canaud L, D'Annoville T, Ozdemir BA, Marty-Ane C, Alric P. Combined endovascular and surgical approach for aortobronchial fistula. J Thorac Cardiovasc Surg 2014;148:2108-11.

5. Canaud L, Ozdemir BA, Bahia S, Hinchliffe R, Loftus I, Thompson M. Thoracic endovascular aortic repair for aortobronchial fistula. Ann Thorac Surg 2013;96:1117-21.

6. Czerny M, Eggebrecht H, Sodeck G, Weigang E, Livi U, Verzini F, et al. New insights regarding the incidence, presentation and treatment options of aorto-oesophageal fistulation after thoracic endovascular aortic repair: the European Registry of Endovascular Aortic Repair Complications. Eur J Cardiothorac Surg 2014;45:452-7.

7. Li M, Langlois N, Byard RW. Fatal aortobronchial fistula. J Forensic Leg Med 2013;20:395-8.

8. Sica G, Rea G, Bocchini G, Lombardi R, Muto M, Valente T. Aortopulmonary fistula presenting without an endoleak after thoracic endovascular aortic repair. Korean J Thorac Cardiovasc Surg 2017;50:287-90.

9. von Segesser LK, Tkebuchava T, Niederhauser U, Kunzli A, Lachat M, Genoni M, et al. Aortobronchial and aortoesopha- geal fistulae as risk factors in surgery of descending thoracic aortic aneurysms. Eur J Cardiothorac Surg 1997;12:195-201.

10. Girdauskas E, Falk V, Kuntze T, Borger MA, Schmidt A, Scheinert D, et al. Secondary surgical procedures after endovascular stent grafting of the thoracic aorta: successful approaches to a challenging clinical problem. J Thorac Cardiovasc Surg 2008;136:1289-94.

11. Prokakis C, Koletsis E, Apostolakis E, Dedeilias P, Dougenis D. Aortoesophageal fistulas due to thoracic aorta aneurysm: surgical versus endovascular repair. Is there a role for combined aortic management? Med Sci Monit 2008;14:RA48-54.

12. Bergqvist D, Bjorck M. Secondary arterioenteric fistulation: a systematic literature analysis. Eur J Vasc Endovasc Surg 2009; 37:31-42.

13. Roselli EE, Abdel-Halim M, Johnston DR, Soltesz EG, Greenberg RK, Svensson LG, et al. Open aortic repair after prior thoracic endovascular aortic repair. Ann Thorac Surg 2014;97: 750-6.

14. Piciche M, De Paulis R, Fabbri A, Chiariello L. Postoperative aortic fistulas into the airways: etiology, pathogenesis, presentation, diagnosis, and management. Ann Thorac Surg 2003; 75:1998-2006.

15. Eggebrecht H, Mehta RH, Dechene A, Tsagakis K, Kuhl H, Huptas S, et al. Aortoesophageal fistula after thoracic aortic stent-graft placement: a rare but catastrophic complication of a novel emerging technique. JACC Cardiovasc Interv 2009;2: $570-6$. 